\title{
Generation of virtual asphalt mixture porosity for computational modelling
}

\author{
A. Chiarelli*, A.R. Dawson, A. García \\ Nottingham Transportation Engineering Centre (NTEC), Faculty of Engineering, The \\ University of Nottingham, University Park, Nottingham, NG7 2RD
}

\begin{abstract}
A new algorithm is proposed for the computational generation of realistic asphalt mixture porosity for computational simulations. The algorithm starts by generating in a $2 \mathrm{D}$ domain a number of randomly positioned circular or elliptical elements that are meant to represent virtual asphalt aggregate particles. These elements are then grown by mimicking the biological mechanism called contact inhibition until a target air voids content (AVC), chosen by the user, is met. In addition, multiple $2 \mathrm{D}$ domains can be converted to $3 \mathrm{D}$ and combined to generate a multi-layered realistic representation of the porosity present in asphalt mixtures. In this paper, the working mechanism of the algorithm is described and its efficiency is assessed. Moreover, the validity of the results is discussed, and virtual domains, in both 2 and 3 dimensions, are compared with real CT scans in order to show the efficacy of this approach. It was found that the virtual representations of the asphalt mixture porosity show realistic characteristics in terms of air voids content and that the air voids size distribution is consistent with that of real specimens.
\end{abstract}

Keywords: asphalt, air voids content, packing, porosity

\footnotetext{
${ }^{*}$ Corresponding author

Email addresses: chiarelli.andrea@gmail.com, +447477570633 (A. Chiarelli), andrew.dawson@nottingham.ac.uk (A.R. Dawson), alvaro.garcia@nottingham.ac.uk (A. García)
} 


\section{Introduction}

Since the properties of asphalt pavements depend on the characteristics of the particles they are made of, the importance of computational particulate models that can describe them is undeniable. In particular, it is interesting to 5 focus on the role of porosity in asphalt pavements, as it influences the strength of the material and its Young's modulus [1. Moreover, in 2, Chen et al. describe the relationship between porosity and fluid flow, showing that also permeability is a function of porosity.

In the literature many examples of computational particulate models can be found, as reported in [3]. The options for the generation of asphalt domains range from simple solutions based on 2D circumferences, to more complex models that imply the use of $3 \mathrm{D}$ rotated ellipsoids: the $2 \mathrm{D}$ option is clearly the less realistic, while the $3 \mathrm{D}$ one requires more powerful computers to be run, thus, the choice between them depends on the final use of the simulations. The existing methods used to generate asphalt models from a particles assemblage usually rely on physical principles, such as inter-particle friction, contact forces, compaction energy, or drop and roll mechanisms [3, 4. Moreover, models based on statistical principles such as Monte Carlo methods can also be used [4].

The creation of specimens using these criteria is complex and requires a clear understanding of the physics behind the processes that are used. For this reason, it is complicated for users to understand clearly the working mechanisms that power such methods, even if their results are reportedly realistic and provide reliable tools for simulations.

The first issue in developing an asphalt assemblage is to select and position the aggregate particles. The problem of generating packed particles in a finite or infinite domain is also of great interest in the fields of physics and mathematics, where it is related to fractal structures [5, 6, 7]. As reported in [8, Apollonian Packing (AP) is the oldest known way to tackle the problem of packing particles in space. The method consists in positioning a new particle (a disk in this case) in the space between three other mutually touching particles, in such a way that 
it touches all of them. This procedure is repeated many times in order to fill all the voids created by the addition of new particles. Apollonian Packing eventually leads to a very dense system with the number of particles approaching infinity, however it does not generate a randomized distribution of particles. As shown in [8], an alternate system was developed to implement a Randomized version of Apollonian Packing (RAP), but also in this case only one new particle is placed each time. This model was generalized to an even greater extent in the ABK model developed by Brilliantov, Krapivsky and Andrienko, where the growth process is called "touch-and-stop model of growth" [9, 10].

40 Another method [7] generates a randomized distribution of packed particles in a volume, but its aim is to simulate the motion of tectonic plates and the appearance of seismic gaps, thus the particles are meant to organize themselves in a system composed of bearings. The structure of the bearings makes it impossible to obtain a realistic organization of the particles for an asphalt sample, as the model is based on the fact that a particle must touch a number of other particles (depending on the geometry, 2D or 3D) to be accepted [7, thus, no porous channel is allowed from and end of a $2 \mathrm{D}$ section of the domain to another, whereas asphalt usually exhibits pore connectivity. Perhaps this limitation could be overcome by the removal or particles from the domain; this, however, would 50 completely change the aim of the model.

\section{Aims}

The objective of this paper is to describe the development of a packing method used to generate asphalt samples for the analysis of the porous space. The method described in this paper is based on purely geometric concepts, without the need of an extensive knowledge of particulate assemblage modeling. The algorithm is able to create $2 \mathrm{D}$ and $3 \mathrm{D}$ domains, in order to allow different kinds of computational analyses. The software developed in the present paper follows the general idea of "touch-and-stop model of growth" 9]. An approach based on randomization in two dimensions is used to obtain a virtual asphalt 
slab with a structure that can be compared to real asphalt samples. The randomization of the parameters of interest enables the creation of a wide range of possible configurations different from one another.

\section{Development of the packing algorithm}

${ }_{65}$ Default mode: 2D packing of circles. In Fig. 1 a flow chart of the algorithm is shown.

The first step in the algorithm is the initialization of the operating variables, which are the size of the domain, the number of centres for the first generation of nucleating particles, the maximum size of the particles, and the target air voids content (AVC). This last parameter is very important for the algorithm, as it is the target to which it iteratively attempts to converge. Let us point out that a difference exists between the real air voids content and the virtually generated one. The assemblage of particles generated by the algorithm in two dimensions does not include the bitumen mastic that fills much of the voids

75 between the aggregate particles in real asphalt. Thus, the value of AVC that is obtained computationally (from now on $A V C^{*}$ ) should be reduced by a factor that takes into account the presence of the binder and the smallest stones, that are smaller in size than the minimum particle diameter permitted in the numerical procedure just described. This factor was chosen as 19\% (7\% binder so $+12 \%$ filler, [1]), thus,

$$
A V C[\%]=A V C^{*}[\%]-19 \%
$$

where $A V C^{*}$ is the air voids content generated by the software and AVC is the real value. As a result, at a computed $A V C^{*}$ equal to $25 \%$, the real $\mathrm{AVC}$ is $6 \%$. Moreover, let us add that the value of the AVC that is calculated by the algorithm is actually a measure of the $2 \mathrm{D}$ void area, thus, it does not correspond 85 to the 3D measure of the void volume.

After the initialization, the algorithm generates a number of particle centres, 


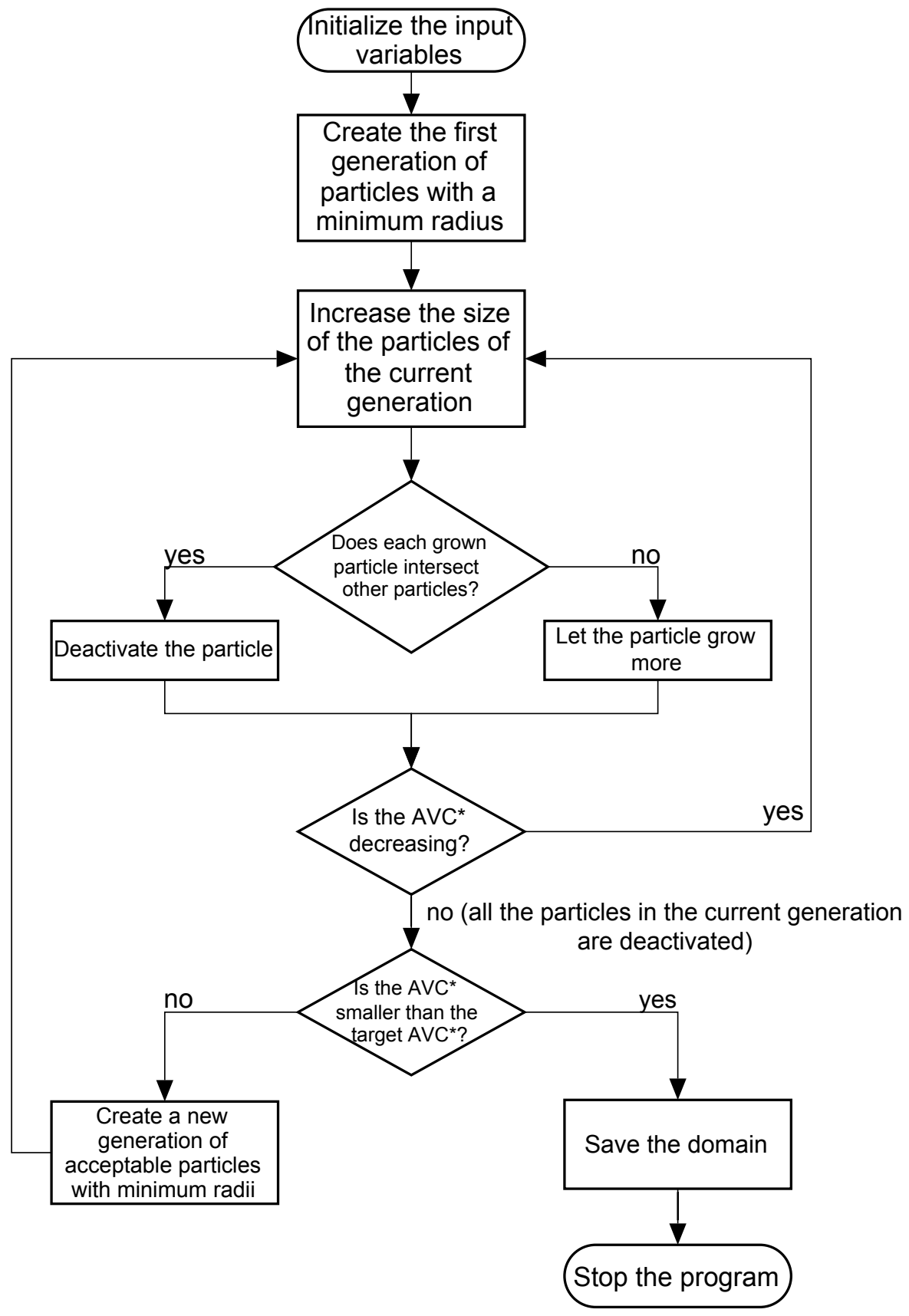

Figure 1: Flowchart of the algorithm ( $A V C^{*}$ indicates the virtual air voids content). 
which are then turned into circumferences with a small radius. These circumferences are then grown according to the parametric equation of a circumference. The circles are grown one by one and the algorithm checks if any intersection between the growing particle and other particles takes place. If the growing circle intersects other particles, it is deactivated and its radius is restored to its previous value, while if there is no intersection the algorithm moves on to another particle and repeats the same procedure.

At some point all the circles will be deactivated once they have all reached their maximum allowed expansion (because they reached a maximum fixed radius or because there is no more space available for their growth): this corresponds to a value of $A V C^{*}$, which is the parameter used to end the current growth loop. The $A V C^{*}$ keeps decreasing while the particles are growing, and it stops decreasing when they are all deactivated. At this point, in order to reach a lower $A V C^{*}$, new particles are needed. Since one of the aims is to guarantee the randomization of the particle distribution, new centres for the new particles are selected in random positions. Of course, many centres will not be in acceptable positions, as they may fall on other particles or be too close to their edges to allow a step of growth. These particles are discarded and not included in the list of particles used for the growth steps that will follow. The new centres go through the same procedure described before, until they are all deactivated and a new generation of centres needs to be generated. After each generation, the current $A V C^{*}$ must be checked to see if it is less than the target $A V C^{*}$. If it is, the procedure is stopped and the generated domain of particles is saved.

2D packing of ellipses. The generation of ellipses requires a further analysis due to the fact that they have axes with different lengths, thus, their geometry can not be so simply described. To begin with, note that two ways are available to generate ellipses: the particles can be generated either with their axes parallel to the axes of the coordinate system or with randomly rotated axes.

The first case is simpler, because the ellipses are defined with their basic para- 


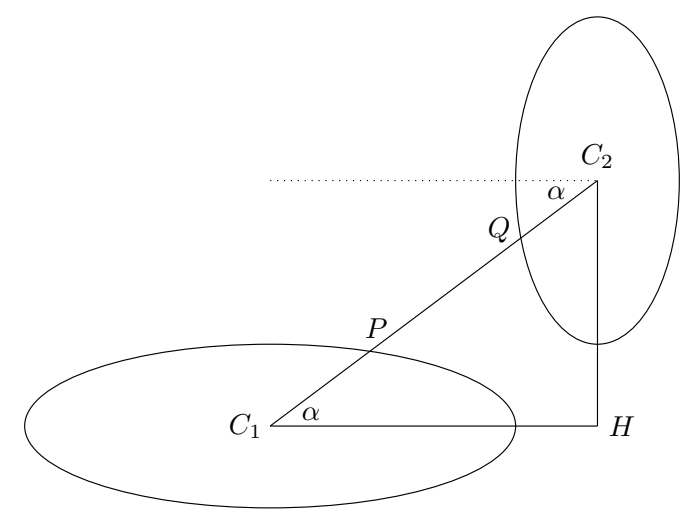

Figure 2: Geometric configuration for growing ellipses.

metric equation

$$
\begin{aligned}
& x=a \cdot \cos (\omega)+c_{x} \\
& y=b \cdot \sin (\omega)+c_{y}
\end{aligned}
$$

where $a$ and $b$ are the major and minor radii of the ellipse, the centre of the particle is $C=\left(c_{x}, c_{y}\right)$, and $\omega$ is a variable ranging from 0 to $2 \pi$. Only the length of the radii and the position of the centres need to be generated. The initial length of the radii can be generated in any chosen way. For example, one may use a randomized length for each radius, a normal distribution with a chosen standard deviation, or fixed values. Another alternative is to determine randomly one of the radii and calculate the length of the other one based on a fixed $a / b$ ratio.

After this step, the procedure that is followed to increase the size of the ellipses is similar to the one used for circles, with the only difference being the way that the distance between the particles is calculated. With reference to Fig. 2, the 
geometric parameters of interest are computed as:

$$
\begin{aligned}
\alpha & =\tan ^{-1}\left[\left|c_{y 1}-c_{y 2}\right| /\left|c_{x 1}-c_{x 2}\right|\right] \\
\overline{C_{1} P} & =\sqrt{\left(a_{1} \cdot \cos (\alpha)\right)^{2}+\left(b_{1} \cdot \sin (\alpha)\right)^{2}} \\
\overline{C_{2} Q} & =\sqrt{\left(a_{2} \cdot \cos (\alpha)\right)^{2}+\left(b_{2} \cdot \sin (\alpha)\right)^{2}} \\
d & =\sqrt{\left(c_{x 1}-c_{x 2}\right)^{2}+\left(c_{y 1}-c_{y 2}\right)^{2}}
\end{aligned}
$$

where $d$ is the distance between the centres and needs to be greater or equal to $\overline{C_{1} P}+\overline{C_{2} Q}$. Every other part in the algorithm follows the mechanism described in the case with circles.

In the case of rotated ellipses, everything becomes more complex, starting with the equations that are used to describe the geometry of the particles:

$$
\begin{aligned}
& x=a \cdot \cos (\omega) \cdot \cos (\theta)-b \cdot \sin (\omega) \cdot \sin (\theta)+c_{x} \\
& y=a \cdot \cos (\omega) \cdot \sin (\theta)+b \cdot \sin (\omega) \cdot \cos (\theta)+c_{y}
\end{aligned}
$$

where $\theta$ is the rotation angle with respect to the horizontal direction. The rotation angle is generated with a normal distribution centred on 0 and with an appropriate standard deviation. As in the previous case with ellipses, the initial length of the radii is determined by the user.

If rotated ellipses are used a problem arises in the computation of their relative distance, because the geometric condition shown in Eq. $3 \mathrm{~d}$ does not work anymore. The reason for this is the fact that the geometric distance is not able to take into account effectively some relative positions between the particles, thus, allowing the generation of intersections. This problem is solved by detecting directly the intersections between growing particles using a MATLAB ${ }^{\circledR}$ function that determines if a point or set of points lies inside a curve.

An example of the creation of a domain of rotated ellipses is shown in Fig. 3, where a generation of new randomly positioned centres (represented by stars) is being placed.

The method for the computation of the real AVC from the virtual $A V C^{*}$ is the 25 same used for circles. 


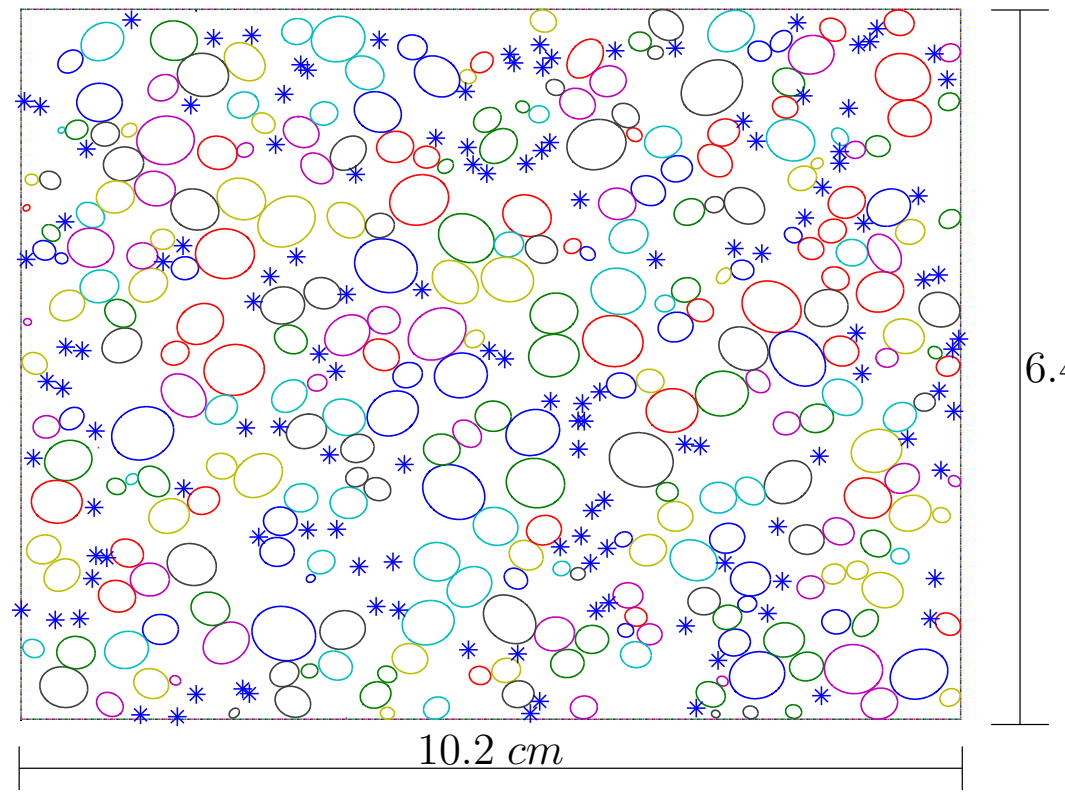

$6.4 \mathrm{~cm}$

Figure 3: Generation of new centres in the case of rotated ellipses.

Generation of $3 D$ pseudo asphalt mixtures. The algorithm is able to convert to $3 \mathrm{D}$ the domains generated in the 2D mode in the cases of circles and ellipses with axes parallel to the main axes. This allows the creation of single layers made of spheres or ellipsoids, where the position of the particles in each 3D layer is determined by their $x$ and $y$ coordinates in the original 2D plane. In the case of ellipsoids, the out-of-plane dimension can be randomized about a mean value with any desired standard deviation or it could be set equal to the major or minor radius of the ellipse.

With a number of $3 \mathrm{D}$ layers it is possible to generate a slab made of previously generated domains laid one on top of another. In order to do this, a first 3D layer is chosen as the bottom of the slab being generated, then new layers are positioned on top of it.

Each additional 3D layer is placed above the bottom layer at an elevation $z$, which is chosen by the user. A graphical representation of the process is shown in Fig. 4. The choice of the elevation allows the generation of 3D domains with 


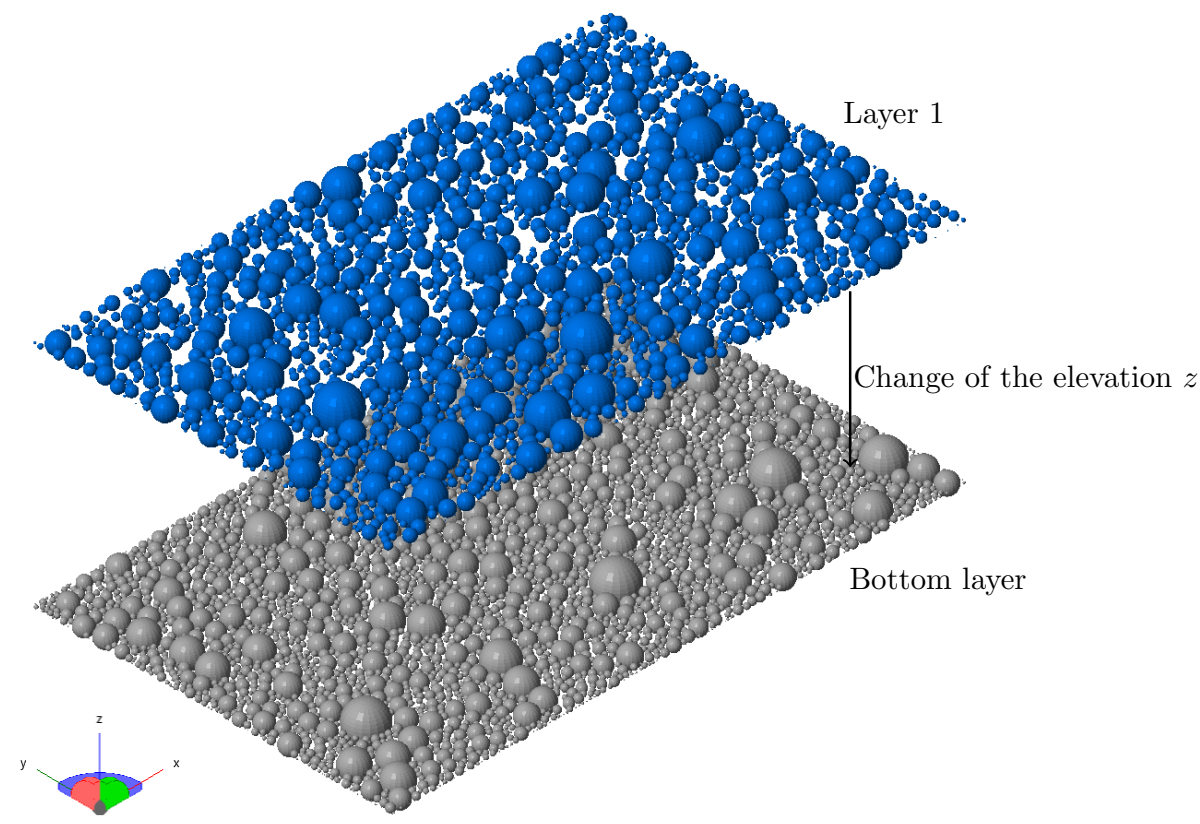

Figure 4: Procedure to generate 3D multi-layered samples with 3D layers converted from 2D packed domains.

different characteristics in terms of air voids content, as it influences how close the 3D layers are placed from one another, thus, it determines how much void space is left between them. So long as the layers are formed from non-identical applications of the $2 \mathrm{D}$ algorithm, there will not be any repeating pattern that could lead to undesirable shapes of the $3 \mathrm{D}$ voids. The flowchart of this procedure is shown in Fig. 5.

Finally, let us add that the AVC is also controlled by choosing the $2 \mathrm{D}$ domains that are converted to 3D and then combined, since the maximum 2D size of the particles (either diameter of the circles or longer radius of the ellipses) highly influences how they are going to intersect in the 3D multi-layered layout.

\section{Implementation of the packing method}

Initiation parameters. Initial values of many of the parameters are needed to allow the process to be applied. The geometric parameters that need to be 


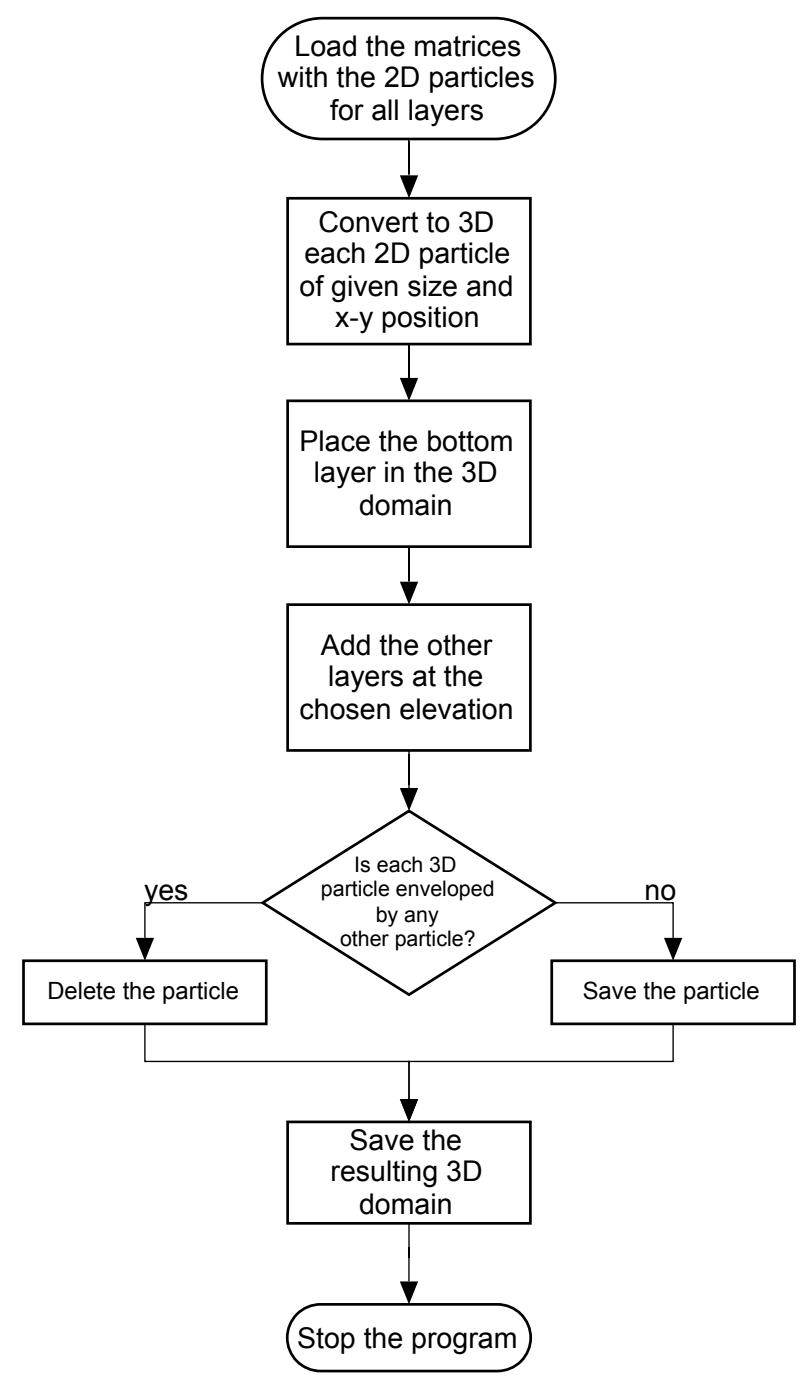

Figure 5: Flowchart of the algorithm for generating 3D slabs.

fixed are the height and width of the domain: as an example, the size of a cross section of a cylindrical asphalt specimen is used, thus having a domain with a height of $102 \mathrm{~mm}$ and a width of $64 \mathrm{~mm}$ (4x2.5 in). A starting population of 500 particles and a maximum diameter of $35 \mathrm{~mm}$ are used in the simulations shown in this paper.

The default value of the initial radius of the circles that are grown is set as 
experienced this slowing as void space becomes increasingly filled. Moreover not only the number of available voids decreases, but also their size becomes increasingly smaller. For this reason, as convergence is approached the collision between newly generated particles becomes unlikely, since they are probably 

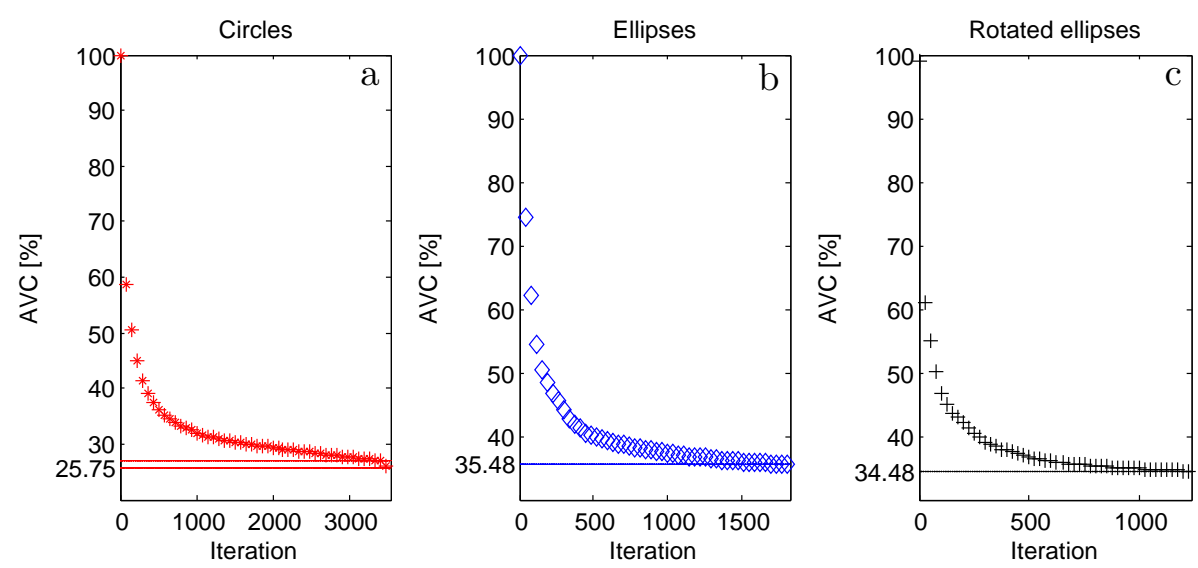

Figure 6: AVC versus time for the cases under investigation.

placed far from one another and between already deactivated (not growing) particles. Eventually there will come a point when the remaining voids between the circles or ellipses cannot be filled with a circle or an ellipse of fixed minimum radius. Thus, a limit for the minimum $A V C^{*}$ exists. Nevertheless, as this limit is approached, disproportionate computational time will be spent by

the algorithm in searching for new possible centres when the empty space is very low and scattered. In order to allow the use of this method for practical purposes, a convergence condition acting as a limit for the execution of the algorithm was chosen. It was first attempted to set as a convergence condition a relative difference in the $A V C^{*}$ of $2.5 E-5$ for two successive iterations. However, the only algorithm that was able to achieve this goal in $2 h$ was the one with circles, while the models with ellipses were stopped when they exceeded the maximum allowed computational time time. This happened because for the ellipses stricter conditions are applied for the acceptance of new centres, thus, less space is filled at every iteration. For this reason, the relative variation of the $A V C^{*}$ for two successive steps is smaller and cannot meet the required condition. Therefore, the limiting condition for ellipses was adapted to the evolution of their $A V C^{*}$ and set as $2 E-4$. It was found that the minimum practical $A V C^{*}$ that could be reached with the mentioned limiting conditions was $27 \%$ 
for the simulation with circles, $35.5 \%$ for the simulation with ellipses, and $34.5 \%$ for the simulation with randomly rotated ellipses. The difference between the two models with ellipses is related to the different ways the shapes occupy space and to the different conditions that are applied for the acceptance of new particles (Euclidean distance vs. MATLAB ${ }^{\circledR}$ function).

Furthermore, the model with rotated ellipses can be run with a rotation angle set equal to zero so as to compare the generation of ellipses in the two ways. As shown in Fig. 7, the use of the model for randomly rotated ellipses to generate ellipses with no rotation provides slower void filling than does the simple ellipse generation method down to $40 \% A V C^{*}$, but past this point the randomly rotated ellipse generation algorithm is clearly more effective. The numbers shown in Fig. 7 are only an example and were obtained as mean values for 100 simulations of each type. However, since the generation of points is randomized with a uniform function, the computational time is always similar.

Domain tracking. Once the model approaches very low values of $A V C^{*}$, and the generation of new particles in random positions has become extremely inefficient, 225 then the randomness of the distribution of particles is already guaranteed. At this stage further reduction of the $A V C^{*}$ can be achieved by simply filling the voids with new particles without the use of a random generator. For this reason, a domain tracker was implemented. The tracking algorithm starts when the $A V C^{*}$ reaches $27 \%$, which had been identified as the minimum practical $A V C^{*}$ for the algorithm generating circles. The line above the minimum $A V C^{*}$ in Fig. 6a corresponds to a value of $27 \%$ and indicates that the next iterations are all performed by the domain tracking algorithm.

The domain tracker creates a grid of points over the generated domain, then it checks if they fall into the empty space or inside any of the circles. If a 235 point of the grid is in the empty space and far enough from any other centre to allow a stage of growth, it is accepted as a new centre, as shown in Fig. 8. When all the points in the grid are checked, their growth starts following the same steps described above. The domain tracking algorithm is run three 


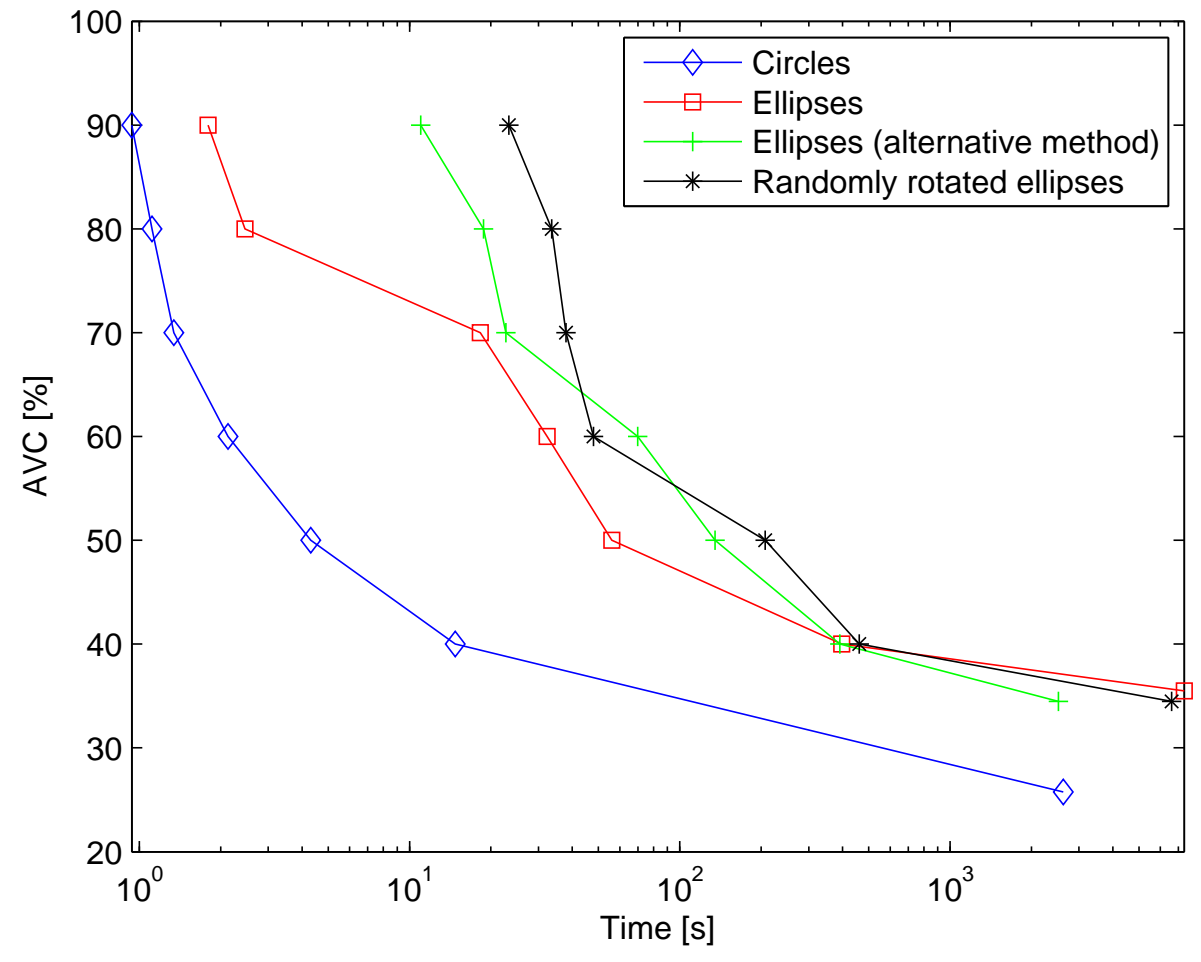

Figure 7: Computational time for the 2D packing models.

times with a shifted grid, thus, many voids can be filled. However, since the domain tracker starts when the algorithm that generates circles has reached an already small $A V C^{*}$, it is clear that the $A V C^{*}$ cannot decrease very much more, since a large part of the domain is already filled. A more dense packing can be achieved by simply increasing the resolution of the grid, which by default is set as $1 E-3$ (this obviously affects the computational time). The value of $1 E-3$ was chosen because a denser grid could lead to a regular arrangement of very tiny particles in the remaining pore space whereas a smaller number of larger, and pseudo-randomly positioned infill particles is required.

Other features of the software. The developed software is able to perform a range of activities based on the data of the generated domain. One feature is 250 the possibility to remove particles from a domain: this is very useful, because 


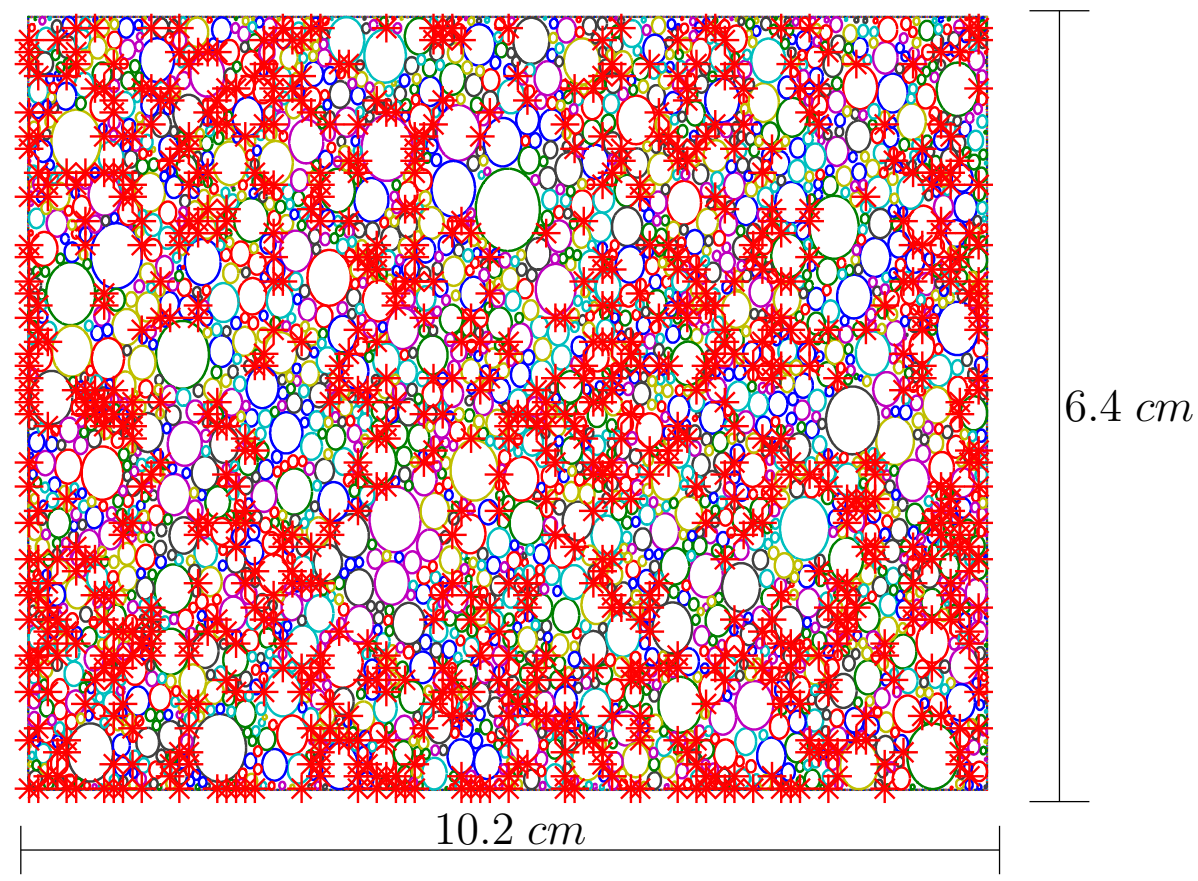

Figure 8: Result of domain tracking in the domain.

the software works with stages of growth and does not stop after every radius increase. For this reason, the removal of particles from the domain allows a direct control of the $A V C^{*}$ by the user, thus, making it possible to obtain intermediate values of $A V C^{*}$. The removing algorithm is again based on the randomization of numbers, i.e., if the user chooses to remove 100 particles, they will be picked randomly from the domain and removed.

\section{Examples of outputs of the algorithm}

In Fig. 9 a virtual cross section generated in 2D (dense domain made of circles) is shown.

260 A number of domains of the kind shown in Fig. 9 can be combined in order to obtain a 3D multi-layered model like the one in Fig. 10. For the analysis of the $3 \mathrm{D}$ samples a clarification is needed. The $3 \mathrm{D}$ domains are meant to represent all the components of the mixture together, as the 3D particles are closely placed 


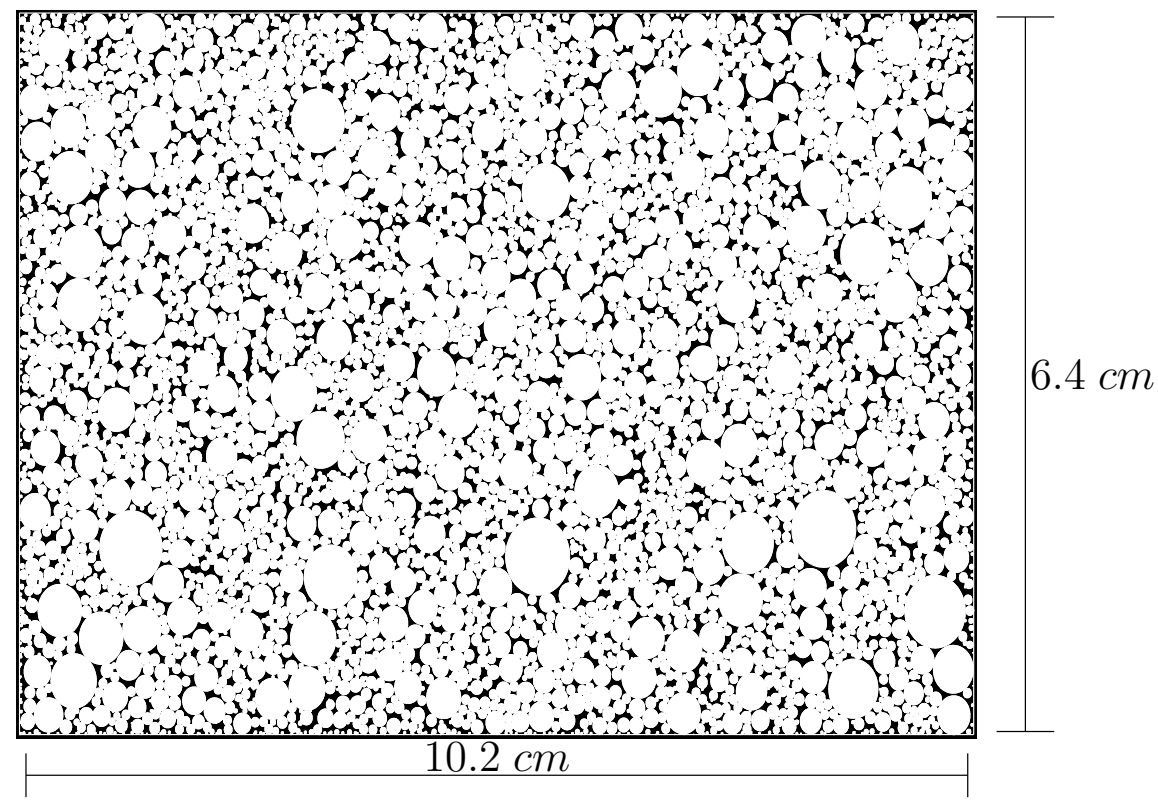

Figure 9: 2D virtual dense asphalt domain (circles), standard size in the software.

and intersect, thus, leaving empty volumes between them: for this reason, the empty volumes are considered as the air voids, while everything else is part of the asphalt mixture. In fact, the $2 \mathrm{D}$ and $3 \mathrm{D}$ domains generated here have a completely different purpose: the bi-dimensional domain allows an analysis of the components of the mixture, while the $3 \mathrm{D}$ model is focused on the generation of voids rather than on the study of the surrounding material.

The 3D model shown in Fig. 10 was generated by trial and error in order to reach a satisfactory result, as further studies are necessary to automate the procedure that links the $2 \mathrm{D}$ air voids content to the corresponding $3 \mathrm{D}$ value. Moreover, the 3D model needs to be repaired after its generation, because the intersection of the particles results in a self-intersecting surface mesh. Finally, let us point out that the extremes of the domain on axis $y$ have to be excluded from the analysis, as they have a very high air void content and possibly an irregular shape due to the 3D repairing procedure. 


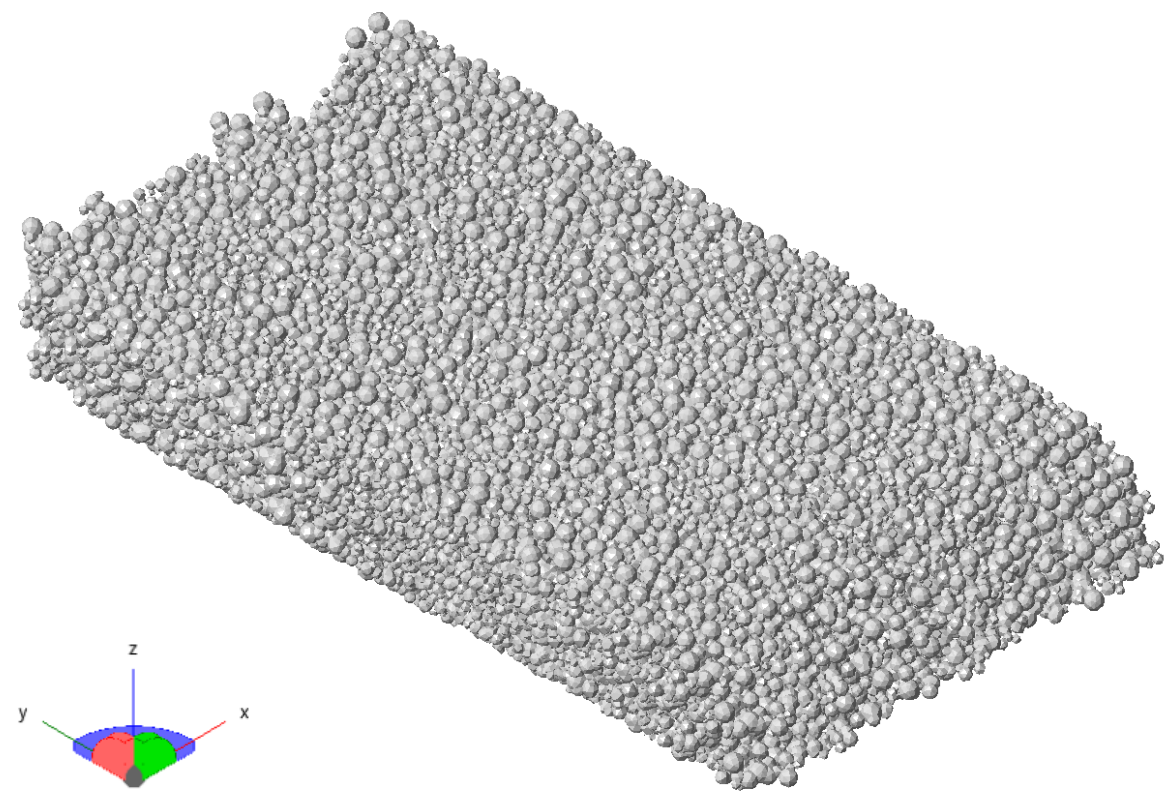

Figure 10: 3D meshed surface of a virtual slab of asphalt.

Mathematical background. Even if the aim of this software is to generate domains for more practical applications, it is very interesting to briefly analyze the

$$
N=N_{0} \cdot n_{b}^{-F D}
$$


where $n_{b}$ is the number of boxes and FD is the fractal dimension, calculated as the local slope of the curve shown. The value of the box-counting dimension is constant in a given interval of box sizes (constant slope), thus, it is possible to say that the domain shows a fractal behaviour for box sizes between $10^{2}$ and $10^{3}$. The fractal dimension in the interval mentioned above is about 1.8 and it is consistent with Liu et Al. [13, where values between 1.63 and 1.82 were found.

The domain that was considered had the minimum value of $A V C^{*}$ it could reach after domain tracking $\left(\sim 25.75 \% A V C^{*}\right)$. The analysis of samples with higher values of $A V C^{*}$ was performed and it showed that the fractal properties were not present: the reason for this is that with high $A V C^{*}$ (e.g., $80 \%$ ) there is no repeating pattern and the domain has large voids.

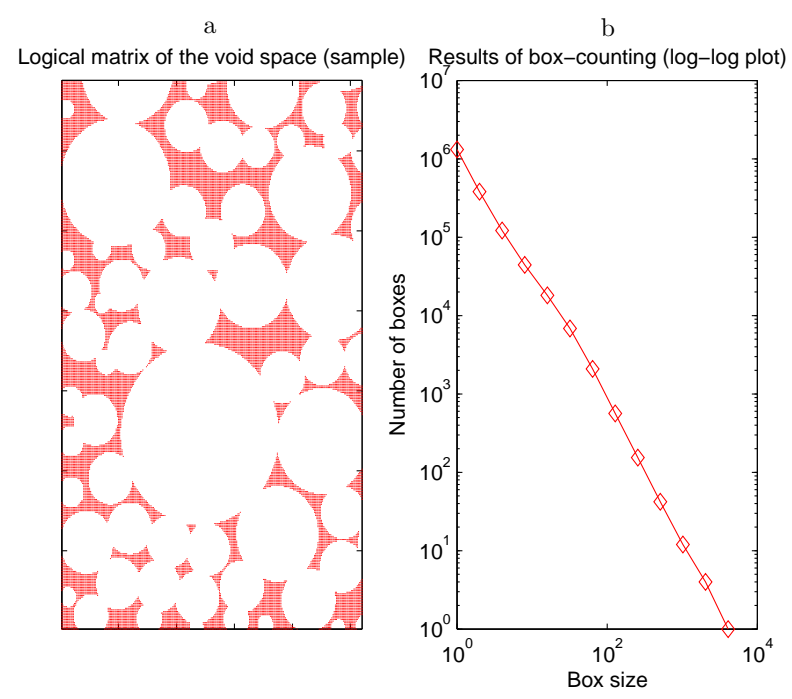

Figure 11: Analysis of the fractal properties of the packed domain.

\section{Comparison with real specimens}

The purpose of the algorithm and its software implementation is to be a tool for computational analyses. For this reason, a comparison with real CT scans of asphalt mixtures in terms of distribution of the air voids is needed to 
confirm the validity of the obtained results. Although direct 3D comparison is conceivable, for reasons of practical convenience 2D CT scan images were compared with 2D cross sections obtained from the generated 3D assemblage described in this paper. Examples of such cross sections are shown in Fig. 12 and Fig. 13, which are the result of the analysis of the 3D domain displayed in Fig. 10. The domain illustrated in Fig. 13 can be used for the analysis of the air voids distribution as follows.

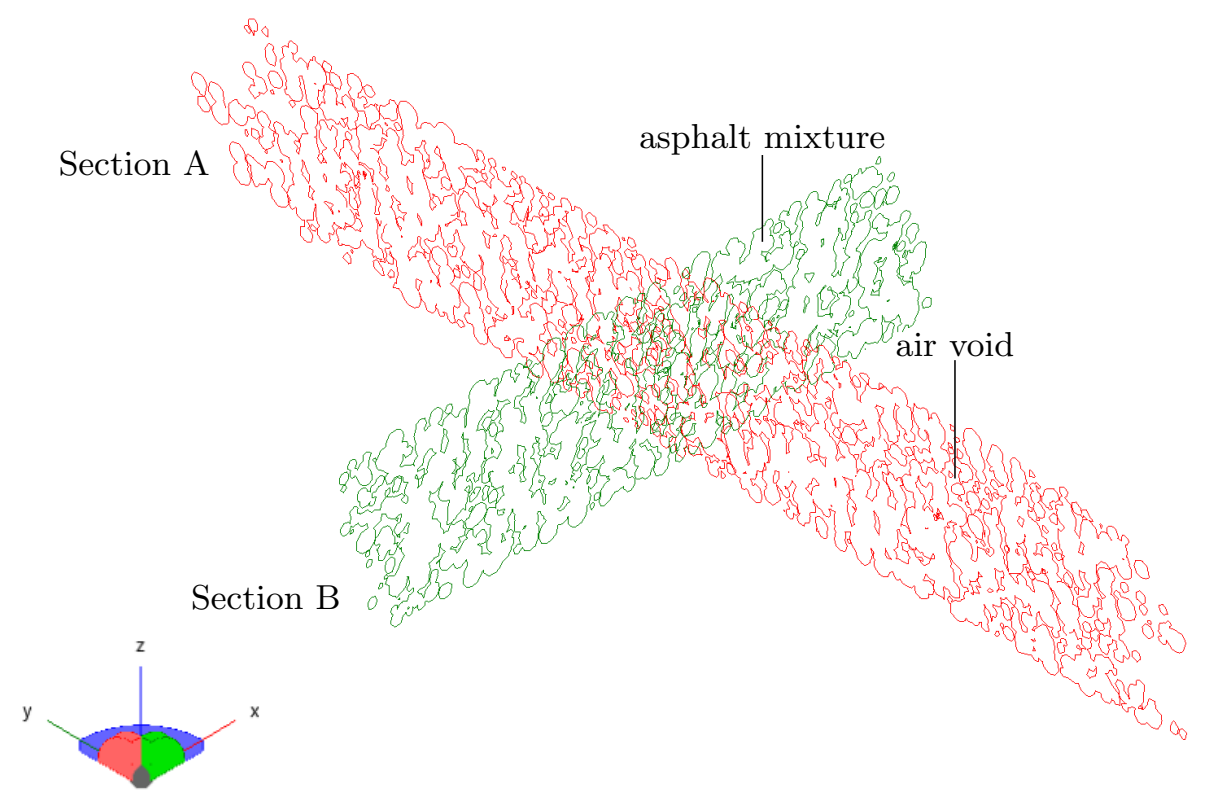

Figure 12: Cross sections on the central axes of the 3D virtual slab shown in Fig. 10.

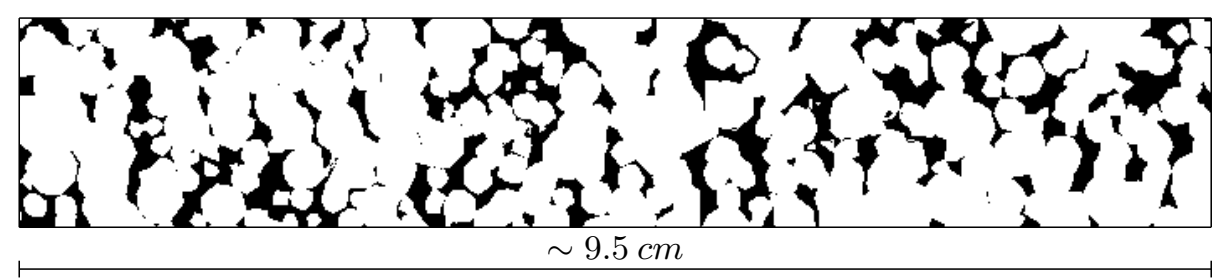

Figure 13: Section B elaborated from Fig. 12, $(A V C=\sim 20.1 \%)$.

In order to perform the comparison between real and virtual samples, the size of the air voids and their distribution were determined for a number of 
cross sections. The CT scans of conventional and porous asphalt used for the comparison are shown in Fig. 14. These binary images were obtained from regular CT scans using a thresholding technique.
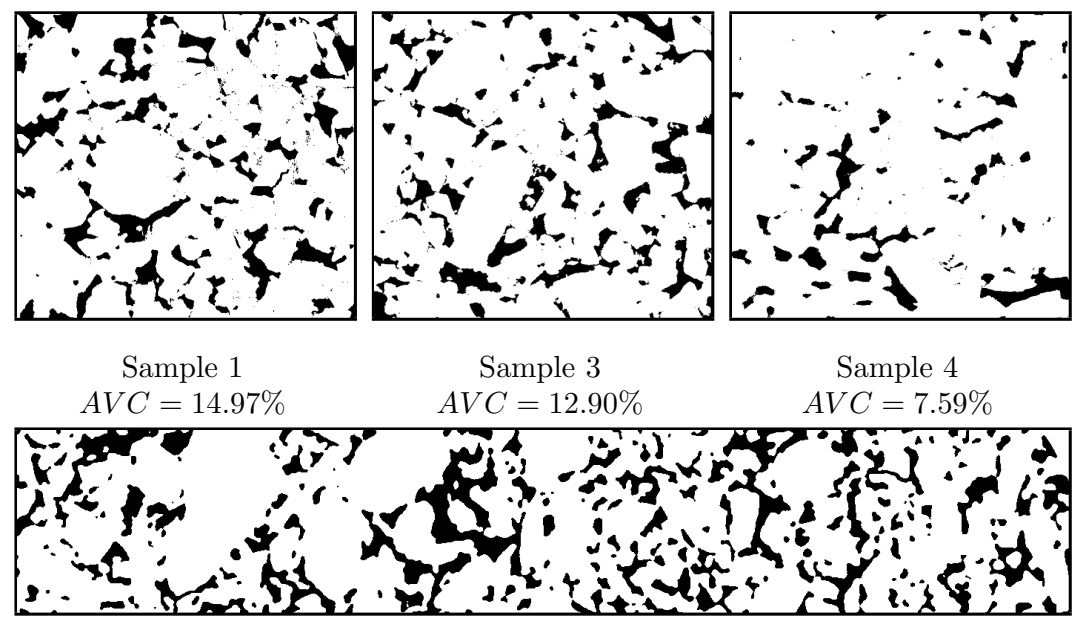

Sample 2

$A V C=22.28 \%$

Figure 14: CT scans used for the comparison with the computationally generated asphalt mixture porosity.

The outliers in the distribution of pores were excluded from each set of values by eliminating the pores with an area with a statistical standard score larger than 3. The standard score can be calculated by subtracting the mean value of a set of data from each element in the set and by dividing the obtained difference by the standard deviation of the data under analysis. The result of this procedure is shown in Fig. 14, where the distribution of the air voids size is represented as a dimensionless quantity on the $x$ axis. In Fig. 15 it is possible to notice that the distribution of the size of the air voids in the virtual specimens shows a realistic behaviour, being encompassed by the other curves. It is interesting to note that certain sized voids are largely missing from the computed assemblage, but that this phenomenon is shared by the scans of genuine asphalt. A plot obtained from a single use of the 2D algorithm described earlier in the paper is also included in Fig. 15. It, too, is encompassed by the 
CT scan curves and, therefore, this suggests that the assembly of a 3D model may not always be necessary where a $2 \mathrm{D}$ analysis of pore structure is required.

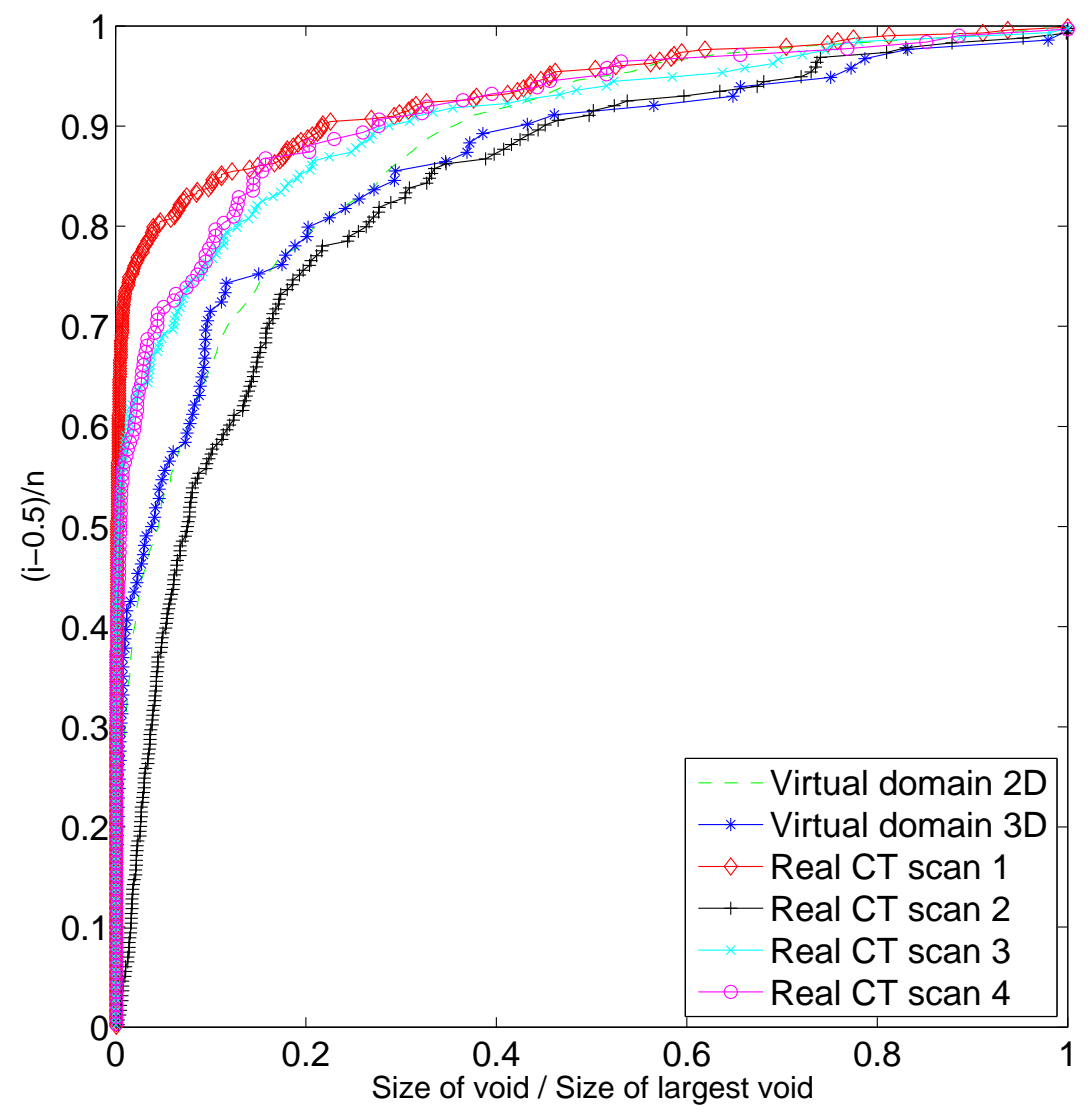

Figure 15: Comparison of the air voids distribution of the virtual samples cross sections with real CT scans.

\section{Applications}

In the field of civil engineering, the algorithm could be used to perform computational analyses of the thermal or mechanical properties of asphalt specimens without the need of making $\mathrm{CT}$ scans, which require time and the proper machinery. In addition, studies about the hydraulic conductivity could be performed as well. 
The major limitation of the algorithm developed is that at the present time it is not able to stack autonomously the 3D planes of particles, therefore this operation needs to be performed with a purposely generated script. Moreover, since the algorithm generates particles, in order to analyse the void space between them the user has to elaborate further the 3D models that are created. This could be avoided by shaping directly the pore structure, as done in other studies [14, 15]. However, the theoretical concepts used in [14] and [15] are more complex than those used in the algorithm developed here, which was studied to simplify the approach to the computational generation of porous media.

Even if the algorithm described here was meant to be used to simulate asphalt specimens, its results can be used for many other applications. The very general approach used allows wide application, although its final purpose could influence strongly its setup and use.

For example, the model could represent particles that can only have a fixed shape due to surface tension, as reported by Andrienko et Al. [10. Another application is obviously in crushed stone, grain, or bead and ball packing problems, where the most efficient way to organize items is investigated. Finally, Brilliantov et Al. 9] state that pattern formation processes are very important for a wide variety of natural phenomena (diffusion-limited aggregation, dendritic growth, dielectric breakdown, etc.), thus, this algorithm and its software implementation could be useful for more theoretical applications linked to the study of repeating structures.

\section{Conclusions}

In this study, a new method to generate virtual asphalt mixture porosity was developed and an analysis of the results was performed. The following conclusions can be drawn:

- The virtual samples generated with this method show realistic characteristics in terms of air voids content and voids distribution, both in two and three dimensions. 


\section{Acknowledgments}

The authors thank the University of Nottingham for the financial support provided for the Ph.D. of Andrea Chiarelli.

\section{References}

[1] M. P. Schpfer, S. Abe, C. Childs, J. J. Walsh, The impact of porosity and crack density on the elasticity, strength and friction of cohesive granular materials: Insights from dem modelling, International Journal of Rock Mechanics \& Mining Sciences 46 (2009) 250-261. doi:10.1016/j.ijrmms. 2008.03 .009 
[2] Y. Chen, C. Shen, P. Liu, Y. Huang, Role of pore structure on liquid flow behaviors in porous media characterized by fractal geometry, Chemical Engineering and Processing 87 (2015) 75-80. doi:10.1016/j.ijrmms.2008. 03.009 .

[3] M. Jiang, J. Konrad, S. Leroueil, An efficient technique for generating homogeneous specimens for DEM studies, Computers and Geotechnics 30 (2003) 579-588. doi:10.1016/S0266-352X (03)00064-8.

[4] R. Isola, Packing of granular materials, Ph.D. thesis, The University of Nottingham (2008).

[5] H. J. Herrmann, G. Mantica, D. Bessis, Space-filling bearings, Physical review letters 65 (1990) 3223-3225. doi:10.1103/PhysRevLett.65.3223

[6] G. Oron, H. J. Herrmann, Generalization of space-filling bearings to arbitrary loop size, Journal of Physics A: Mathematical and General 33 (2000) 1417-1422. doi:10.1088/0305-4470/33/7/310.

[7] R. M. Baram, H. J. Herrmann, Random bearings and their stability, 410 \ Physical Review Letters 95 (2005) 224303-1-224303-4. doi:10.1103/ PhysRevLett.95.224303.

[8] G. W. Delaney, S. Hutzler, T. Aste, Relation between grain shape and fractal properties in random apollonian packing with grain rotation, Physical Review Letters 101 (2008) 120602-1-120602-4. doi:10.1103/ PhysRevLett.101.120602.

[9] N. V. Brilliantov, P. L. Krapivsky, Y. A. Andrienko, Random space-fillingtiling: fractal properties and kinetics, Journal of Physics A: Mathematical and General 27 (1994) L381. doi:10.1088/0305-4470/27/11/006.

[10] Y. A. Andrienko, N. V. Brilliantov, P. L. Krapivsky, Pattern formation by growing droplets: The touch-and-stop model of growth, Journal of Statistical Physics 75. doi:10.1007/BF02186870. 
[11] J. Rebbechi, Stone Mastic Asphalt - Design \& Application Guide, Australian Asphalt Pavement Association (2000).

[12] P. S. Dodds, J. S. Weitz, Packing-limited growth, Physical Review E 65 (2002) 056108-1-056108-6. doi:10.1103/PhysRevE.65.056108.

[13] T. Liu, X. Zhang, Z. Li, Z. Chen, Research on the homogeneity of asphalt pavement quality using x-ray computed tomography (ct) and fractal theory, Construction and Building Materials 68 (2014) 592-593. doi:10.1016/j . conbuildmat.2014.06.046.

[14] M. Siena, M. Riva, J. D. Hyman, C. L. Winter, A. Guadagnini, Relationship between pore size and velocity probability distributions in stochastically

1 $\quad$ generated porous media, Physical Review E 89 (2014) 013018. doi:10. 1103/PhysRevE.89.013018

[15] J. D. Hyman, P. K. Smolarkiewicz, C. L. Winter, Pedotransfer functions for permeability: A computational study at pore scales, Water Resources Research 49 (2013) 2080-2092. doi:10.1002/wrcr.20170. 